03

\title{
О бародиффузии при медленных течениях газовой смеси
}

\author{
(C) В.М. Жданов \\ Национальный исследовательский ядерный университет „МИФИ“, \\ 115409 Москва, Россия \\ e-mail:zhdanov@plasma.mephi.ru
}

Поступило в Редакцию 21 июля 2018 г.

В окончательной редакции 2 ноября 2018 г.

Принято к публикации 7 декабря 2018 г.

\begin{abstract}
Бародиффузия при медленных течениях газовой смеси исследована на основе приближения, использующего гидродинамические уравнения движения для отдельных компонентов смеси. Показано, что учет вязкого переноса импульса и вклада кнудсеновских слоев при течении смеси в канале оказывают заметное влияние на величину бародиффузионного фактора. Получены выражения для усредненных диффузионных потоков компонентов и полного потока смеси в круглом цилиндрическом капилляре, справедливые для умеренно малых чисел Кнудсена, которые использованы для расчета диффузионного бароэффекта и эффекта разделения при течении газовой смеси в системе капилляров, соединяющих два объема. Модификация выражений для бародиффузионного фактора (и перекрестного по отношению к нему коэффициента диффузионного скольжения) позволила объяснить экспериментально наблюдаемую смену знака этих эффектов для некоторых смесей газов при промежуточных значениях числа Кнудсена.
\end{abstract}

DOI: $10.21883 / J T F .2019 .05 .47463 .288-18$

\section{Введение}

Проблема бародиффузии (диффузии под действием градиента давления) давно обсуждается в научной литературе [1-4]. Оценка влияния бародиффузии оказывается важной при анализе эффектов разделения газовых смесей в звуковых и ударных волнах [5-7], радиального разделения в газовой и плазменной центрифугах $[8,9]$, эффекта „масс-диффузии“ (разделения компонентов газовой смеси в присутствии третьего буферного газа [4]) и в ряде других случаев. Особое место занимают исследования диффузионных явлений при медленных течениях газовых смесей через систему тонких капилляров либо пористую мембрану [10-12]. Изменение состава смеси при течении через пористую среду под действием приложенной разности давлений (эффект разделения) лежит, как известно, в основе промышленного диффузионного метода обогащения урана $[13,14]$. К рассматриваемой проблеме тесно примыкают также исследования перекрестного по отношению к эффекту разделения диффузионного бароэффекта [15-17]. Анализ этих эфектов позволяет, в частности, из сравнения экспериментальных и теоретических результатов определять значения параметров, характеризующих взаимодействия молекул со стенкой для компонентов газовой смеси.

Общее феноменологическое выражение для диффузионного потока в смеси, включающего, наряду с обычной (концентрационной) диффузией, также бародиффузию и термодиффузию, можно получить, используя методы неравновесной термодинамики (НT) [2,18]. При этом коэффициент бародиффузии в отличие от термодиффузии оказывается выраженным только через термодинамические параметры смеси [2]. Вместе с тем еще в 1962 г. в работе [3] была выдвинута идея (Ю. Каган) о том, что в число независимых термодинамических сил в линейных соотношениях НТ для потоков, наряду с градиентами обычных термодинамических переменных, могут входить величины соответствующей тензорности, получающиеся дифференцированием неравновесных потоков по координатам. В частности, при установившемся вязком течении смеси учет производной от тензора вязких напряжений в выражении для диффузионного потока приводит к переопределению коэффициента бародиффузии, который становится существенно кинетической величиной.

Строгое описание диффузии в газовой смеси связано, как известно, с решением кинетического уравнения Больцмана на основе хорошо развитых приближенных методов (метод Чепмена-Энскога [1], метод моментов Грэда $[19,3])$. В первом из них, который является методом возмущений по малому параметру (числу Кнудсена), уравнения так называемого первого приближения преобразуются с помощью уравнений сохранения нулевого приближения (уравнений Эйлера), записываемых в пренебрежении всеми диссипативными членами, связанными с вязкостью и теплопроводностью в газовой смеси. По этой причине в первом приближении метода отсутствует влияние вязкого переноса импульса на диффузию в газовой смеси, которое проявляется при использовании лишь следующего (барнеттовского) приближения, требующего довольно громоздких вычислений [1]. Между тем этот эффект, как было показано впервые в [3], сразу выявляется при последовательном использовании метода моментов Грэда.

В настоящей работе для исследования бародиффузии мы воспользуемся достаточно простым и эффективным методом получения выражений для диффузионных потоков, основанном на уравнении баланса импульса отдель- 
ного компонента смеси, которое следует непосредственно из кинетического уравнения Больцмана. Получаемое при этом уравнение, как было показано в [20,21], может быть преобразовано к виду, близкому по структуре к обычному гидродинамическому уравнению движения газа в целом, но записываемому для компонента смеси. Принятый подход, называемый иногда гидродинамическим $[21,22]$, позволяет получить не только известные выражения кинетической теории с классическими значениями коэффициентов диффузии и бародиффузии, но и обосновать модификацию последнего в случае медленных (вязких) течений газовой смеси. Более того, анализ течений смеси в каналах при умеренно малых числах Кнудсена показывает, что в усредненных по сечению канала диффузионных потоках компонентов возникает дополнительный вклад в бародиффузионный фактор, связанный с учетом поведения функции распределения молекул в тонких кнудсеновских слоях на границе газа и стенки.

В качестве примера в настоящей работе рассматривается получение выражений для диффузионных потоков компонентов и полного потока смеси в круглом цилиндрическом капилляре, которые могут служить основой для расчета диффузионного бароэффекта и эффекта разделения при течении газовой смеси в системе капилляров, соединяющих два объема. Модификация выражений для бародиффузионного фактора (и перекрестного по отношению к нему коэффициента диффузионного скольжения) позволяет при этом объяснить экспериментально наблюдаемую смену знака этих эффектов для некоторых выбранных смесей газов при промежуточных значениях числа Кнудсена.

\section{Гидродинамическая теория диффузии}

Напомним сначала известный результат, следующий из строгой кинетической теории газовых смесей [1]. Введем определение для молярного диффузионного потока компонента $\alpha$ в смеси

$$
\mathbf{J}_{\alpha}^{m}=n_{\alpha}\left(\mathbf{u}_{\alpha}-\mathbf{u}^{m}\right),
$$

где $n_{\alpha}-$ числовая плотность, $\mathbf{u}_{\alpha}-$ макроскопическая скорость компонента, $\mathbf{u}^{m}=\sum_{\alpha} x_{\alpha} \mathbf{u}_{\alpha}-$ среднемолярная скорость смеси, $x_{\alpha}=n_{\alpha} / n-$ относительная молярная концентрация (молярная доля) компонента смеси. В случае бинарной газовой смеси имеем

$$
\mathbf{J}_{1}^{m}=n_{1}\left(\mathbf{u}_{1}-\mathbf{u}^{m}\right)=n x_{1} x_{x}\left(\mathbf{u}_{1}-\mathbf{u}_{2}\right), \mathbf{J}_{2}^{m}=-\mathbf{J}_{1}^{m} .
$$

Выражение для $\mathbf{J}_{1}^{m}$, получаемое в рамках первого приближения метода Чепмена-Энскога (без учета термодиффузии), записывается в виде [1]:

$$
\mathbf{J}_{1}^{m}=-n\left[D_{12}\right]_{1}\left[\nabla x_{1}+\alpha_{p}^{m} x_{1} x_{2} \frac{1}{p} \nabla p-\frac{\rho_{1} \rho_{2}}{p \rho}\left(\frac{\mathbf{F}_{1}}{m_{1}}-\frac{\mathbf{F}_{2}}{m_{2}}\right)\right] .
$$

Здесь $\rho_{\alpha}=m_{\alpha} n_{\alpha}-$ массовая плотность компонента, $n=n_{1}+n_{2} \quad$ и $\rho=\rho_{1}+\rho_{2}-$ числовая и массовая плотности смеси, $p$ - давление, $\left[D_{12}\right]_{1}-$ коэффициент взаимной диффузии частиц (первое приближение Чепмена-Каулинга [1]), $\mathbf{F}_{\alpha}$ - внешняя сила, действующая на частицу сорта $\alpha$. Коэффициент $\alpha_{p}^{m}$ носит название бародиффузионного фактора и определяется как

$$
\alpha_{p}^{m}=\frac{m_{2}-m_{1}}{\bar{m}},
$$

где $\bar{m}=\rho / n=m_{1} x_{1}+m_{2} x_{2}-$ средняя масса.

Покажем теперь, при каких условиях этот же результат можно получить исходя из гидродинамических уравнений движения компонентов смеси. Общее уравнение движения смеси в гидродинамике записывается, как известно, в виде [2,18]:

$$
\rho \frac{d \mathbf{u}}{d t}+\nabla p+\nabla \hat{\boldsymbol{\pi}}-\sum_{\alpha} n_{\alpha} \mathbf{F}_{\alpha}=0 .
$$

Здесь $d / d t=\partial / \partial t+(\mathbf{u} \cdot \nabla)-$ оператор полной производной по времени, $\mathbf{u}=\rho^{-1} \sum_{\alpha} \rho_{\alpha} \mathbf{u}_{\alpha}-$ среднемассовая скорость смеси, $\hat{\boldsymbol{\pi}}-$ тензор вязких напряжений в смеси. Величина $\left(\nabla \hat{\boldsymbol{\pi}}_{i}\right)=\partial \pi_{i k} / \partial x_{k}$ соответствует $i$-й компоненте вектора силы вязкого трения, действующей на единицу объема смеси. Соответствующее уравнение движения для отдельного компонента смеси можно представить как [20,21]:

$$
\rho_{\alpha} \frac{d_{\alpha} \mathbf{u}_{\alpha}}{d t}+\nabla p_{\alpha}+\nabla \hat{\boldsymbol{\pi}}_{\alpha}-n_{\alpha} \mathbf{F}_{\alpha}=\mathbf{R}_{\alpha}
$$

где $d_{\alpha} / d t=\partial / \partial t+\left(\mathbf{u}_{\alpha} \cdot \nabla\right), p_{\alpha}-$ парциальное давление и $\hat{\boldsymbol{\pi}}_{\alpha}-$ парциальный тензор вязких напряжений. При записи (6) опущен малосущественный член вида $\nabla \rho_{\alpha} \mathbf{w}_{\alpha} \mathbf{w}_{\alpha}$, квадратичный относительно малых (по сравнению со средней тепловой скоростью частиц) диффузионных скоростей $\mathbf{w}_{\alpha}=\mathbf{u}_{\alpha}-\mathbf{u}$. При сохранении этого члена суммирование уравнений (6) по индексу $\alpha$ при выполнении дополнительного условия $\sum_{\alpha} \mathbf{R}_{\alpha}=0$ приводит к точному уравнению движения смеси (5). Величина $\mathbf{R}_{\alpha}$ в правой части уравнения (6) соответствует средней передаче импульса при столкновениях частиц различного сорта и носит название „диффузионной силы трения“". Простые соображения, основанные на введении средней или эффективной частоты столкновений $\bar{\nu}_{\alpha \beta}$ для частиц сортов $\alpha$ и $\beta$, приводят к выражению для $\mathbf{R}_{\alpha}$ вида $[21,22]$ :

$$
\mathbf{R}_{\alpha}=-\sum_{\beta \neq \alpha}^{N} n_{\alpha} \mu_{\alpha \beta} \bar{v}_{\alpha \beta}\left(\mathbf{u}_{\alpha}-\mathbf{u}_{\beta}\right),
$$

где $\mu_{\alpha \beta}=m_{\alpha} m_{\beta} /\left(m_{\alpha}+m_{\beta}\right)-$ приведенная масса частиц, $N$ - число компонентов смеси. Другое более удобное представление $\mathbf{R}_{\alpha}$ связывает его с коэффициентом бинарной диффузии частиц $\left[D_{\alpha \beta}\right]_{1}$, так, что $[21]$ :

$$
\mathbf{R}_{\alpha}=-\sum_{\beta \neq \alpha}^{N} \frac{n_{\alpha} n_{\beta} k T}{n\left[D_{\alpha \beta}\right]_{1}}\left(\mathbf{u}_{\alpha}-\mathbf{u}_{\beta}\right) .
$$


Заменим производную $d_{\alpha} \mathbf{u}_{\alpha} / d t$ в левой части уравнения (6) на $d \mathbf{u} / d t$, что соответствует пренебрежению членами порядка $d \mathbf{w}_{\alpha} / d t$ по сравнению с членами порядка $\bar{v}_{\alpha \beta} \mathbf{w}_{\alpha}$ в правой части (6). Последнее согласуется с обычно принимаемым в теории явлений переноса в газе предположением, что макроскопические параметры смеси слабо меняются за времена и на расстояниях порядка средних характерных времени и длины свободного пробега частиц между столкновениями. Вместо уравнения (6) тогда имеем

$$
\rho_{\alpha} \frac{d \mathbf{u}}{d t}+\nabla p_{\alpha}+\nabla \hat{\boldsymbol{\pi}}_{\alpha}-n_{\alpha} \mathbf{F}_{\alpha}=-\sum_{\beta \neq \alpha}^{N} \frac{n_{\alpha} n_{\beta} k T}{n\left[D_{\alpha \beta}\right]_{1}}\left(\mathbf{u}_{\alpha}-\mathbf{u}_{\beta}\right) .
$$

Рассмотрим случай, когда в газе пренебрегается необратимым переносом импульса, связанным с наличием внутреннего трения (вязкости). В уравнениях (5) и (6) можно тогда опустить члены $\nabla \hat{\boldsymbol{\pi}}$ и $\nabla \hat{\boldsymbol{\pi}}_{\alpha}$. Уравнение (5) соответствует в этом случае уравнению Эйлера для идеальной жидкости [2]. Выражая из этого уравнения производную $d \mathbf{u} / d t$ и подставляя ее в уравнение (9), можно представить последнее в виде

$$
p \mathbf{d}_{\alpha}=-\sum_{\beta \neq \alpha}^{N} \frac{n_{\alpha} n_{\beta} k T}{n\left[D_{\alpha \beta}\right]_{1}}\left(\mathbf{u}_{\alpha}-\mathbf{u}_{\beta}\right),
$$

где величина

$$
p \mathbf{d}_{\alpha}=\left(\nabla p_{\alpha}-\frac{\rho_{\alpha}}{\rho} \nabla p\right)-\left(n_{\alpha} \mathbf{F}_{\alpha}-\frac{\rho_{\alpha}}{\rho} \sum_{\beta} n_{\beta} \mathbf{F}_{\beta}\right)
$$

называется термодинамической диффузионной силой. Уравнение (10) известно в литературе по кинетической теории газов как уравнение Стефана-Максвелла, описывающее изотермическую диффузию в многокомпонентной газовой смеси [23].

Легко обнаружить, что в случае бинарной смеси из уравнения (10) с учетом (11) мы получаем приведенный выше результат для $\mathbf{J}_{1}^{m}(3)$, следующий из строгой кинетической теории [1], с бародиффузионным фактором в форме (4).

Обратим внимание на то, что полученный нами результат справедлив только для инерционных (невязких) течений газовой смеси. Отметим также, что для так называемых массовых сил, пропорциональных первой степени масс частиц (например, сила тяжести, силы инерции), последний член в правой части выражения (3), пропорциональный разности ускорений, обращается в нуль, т.е. диффузионный поток определяется тем же выражением, что и в отсутствие внешних сил. Бародиффузионный фактор $\alpha_{p}$ также сохраняет при этом свое обычное значение (4). При воздействии на частицы смеси внешних сил другой природы возможна форма записи (3) с эффективным бародиффузионным фактором $\alpha_{p}^{*}$, который может заметно отличаться от (4). Этот вопрос подробно обсуждался в $[4,24]$.
Легко обнаружить, что выражение для $\alpha_{p}^{m}$ (4) согласуется также с результатом, получаемым методами неравновесной термодинамики [2,25]. При этом следует иметь в виду, что в традиционной схеме НТ $[2,18]$ вместо молярных диффузионных потоков оперируют обычно с массовыми диффузионными потоками $\mathbf{J}_{\alpha}=\rho_{\alpha}\left(\mathbf{u}_{\alpha}-\mathbf{u}\right)$, которые определяются в системе отсчета, связанной со среднемассовой скоростью и. Для изотермического течения бинарной смеси эти потоки (в отсутствие внешних сил) определяются выражениями [2]:

$$
\mathbf{J}_{1}=-\rho D\left(\nabla c_{1}+\alpha_{p} c_{1} c_{2} \frac{1}{p} \nabla p\right), \mathbf{J}_{2}=-\mathbf{J}_{1},
$$

где $c_{\alpha}=\rho_{\alpha} / \rho$ - относительная массовая доля компонента $\alpha$ смеси. Потоки $\mathbf{J}_{1}$ и $\mathbf{J}_{1}^{m}$, а также концентрации $c_{\alpha}$ и $x_{\alpha}$, связаны соотношениями [22,25]:

$$
\mathbf{J}_{1}=\frac{\rho c_{1} c_{2}}{n x_{1} x_{2}} \mathbf{J}_{1}^{m}, \quad c_{\alpha}=\frac{m_{\alpha}}{\bar{m}} x_{\alpha} .
$$

Сравнение соответствующих форм записи для $\mathbf{J}_{1}^{m}$ и $\mathbf{J}_{1}$ [25] показывает, что при этом выполнены условия $D=D_{12}, \alpha_{p}=\alpha_{p}^{m}$.

Выражение для $\alpha_{p}$ в случае бинарной газовой смеси идеальных газов, рассчитанное методами НТ в [2], совпадает с выражением (4):

$$
\alpha_{p}=\left(m_{2}-m_{1}\right)\left(\frac{c_{1}}{m_{1}}+\frac{c_{2}}{m_{2}}\right)=\frac{m_{2}-m_{1}}{\bar{m}} .
$$

\section{Влияние вязкого переноса импульса на диффузию}

Рассмотрим теперь другой важный случай, который соответствует установившемуся вязкому течению газовой смеси. В этом случае в левых частях уравнений (5) и (9) можно опустить инерционные члены с производной $d \mathbf{u} / d t$ в силу их малости по сравнению с членами, описывающими вязкий перенос импульса, что соответствует медленным течениям газовой смеси (малые числа Рейнольдса). Полагая для простоты, что внешние силы отсутствуют, находим, что градиент давления уравновешивается в этом случае силами вязкого трения, действующими в газовой смеси:

$$
\nabla p+\nabla \hat{\boldsymbol{\pi}}=0 .
$$

Уравнение баланса импульса для отдельного компонента смеси (6) принимает вид

$$
\nabla p_{\alpha}+\nabla \hat{\boldsymbol{\pi}}_{\alpha}=-\sum_{\beta \neq \alpha}^{N} \frac{n_{\alpha} n_{\beta} k T}{n\left[D_{\alpha \beta}\right]_{1}}\left(\mathbf{u}_{\alpha}-\mathbf{u}_{\beta}\right) .
$$

Для определения $\hat{\boldsymbol{\pi}}_{\boldsymbol{\alpha}}$ и $\hat{\boldsymbol{\pi}}$ можно воспользоваться известными результатами кинетической теории, получаемыми, например, на основе первого приближения метода Чепмена-Энскога [1], из которых следуют линейные соотношения для тензоров вязких напряжений вида

$$
\pi_{\alpha i k}=-2 \eta_{\alpha} \varepsilon_{i k}, \pi_{i k}=-2 \eta \varepsilon_{i k},
$$


где $\eta_{\alpha}$ и $\eta=\sum_{\alpha} \eta_{\alpha}-$ парциальный и полный коэффициенты вязкости соответственно, а $\varepsilon_{i k}-$ тензор скоростей сдвига

$$
\varepsilon_{i k}=\frac{1}{2}\left(\frac{\partial u_{i}}{\partial x_{k}}+\frac{\partial u_{k}}{\partial x_{i}}\right)-\frac{1}{3} \delta_{i k} \nabla \cdot \mathbf{u} .
$$

Используя (13) и соотношения (15), находим

$$
\frac{\partial \varepsilon_{i k}}{\partial x_{k}}=\frac{1}{2 \eta} \frac{\partial p}{\partial x_{i}}, \frac{\partial \pi_{\alpha i k}}{\partial x_{s}}=-2 \eta_{\alpha} \frac{\partial \varepsilon_{i k}}{\partial x_{k}}=-\frac{\eta_{\alpha}}{\eta} \frac{\partial p}{\partial x_{i}} .
$$

В результате уравнение (14) можно представить в виде

$$
p \mathbf{d}_{\alpha}^{v}=-\sum_{\alpha \neq \beta}^{N} \frac{n_{\alpha} n_{\beta} k T}{n\left[D_{\alpha \beta}\right]_{1}}\left(\mathbf{u}_{\alpha}-\mathbf{u}_{\beta}\right)
$$

где

$$
p \mathbf{d}_{\alpha}^{v}=\nabla p_{\alpha}-\frac{\eta_{\alpha}}{\eta} \nabla p=\left[\nabla x_{\alpha}+\left(x_{\alpha}-\frac{\eta_{\alpha}}{\eta}\right) \frac{1}{p} \nabla p\right] .
$$

Для случая бинарной смеси газов из уравнения (16) следует

$$
\mathbf{J}_{1}^{m}=-n\left[D_{12}\right]_{1}\left(\nabla x_{1}+\left[\alpha_{p}^{\nu}\right]_{1} x_{1} x_{2} \frac{1}{p} \nabla p\right),
$$

где $\left[\alpha_{p}^{\nu}\right]_{1}-$ бародиффузионный фактор в вязком потоке смеси (первое приближение), который определяется выражением

$$
\left[\alpha_{p}^{\nu}\right]_{1}=\frac{1}{x_{1} x_{2}}\left(x_{1}-\frac{\eta_{1}}{\eta}\right)=\frac{1}{\eta}\left(\frac{\eta_{2}}{x_{2}}-\frac{\eta_{1}}{x_{1}}\right) .
$$

Выражения для парциальных коэффициентов вязкости $\eta_{\alpha}$ (не путать с вязкостью чистого газа из частиц сорта $\alpha$ ), соответствующих первому приближению Чепмена-Каулинга, можно найти, например, в [3,21]. Отметим, что в отличие от коэффициента $\alpha_{p}$ (4) бародиффузионный фактор в вязком потоке $\left[\alpha_{p}^{\nu}\right]_{1}$ оказывается существенно кинетической величиной, зависящей, помимо масс и концентраций компонентов, от эффективных сечений столкновений частиц смеси.

Выражение $\left[\alpha_{p}^{\nu}\right]_{1}$ для в виде (19) было получено более сложным путем в [3] на основе приближения десяти моментов метода Грэда, используемого для решения кинетического уравнения Больцмана для смеси газов. Там же приводится результат, соответствующий более полному приближению тринадцати моментов. При этом коэффициент бинарной диффузии $\left[D_{12}\right]_{1}$ в выражении для $\mathbf{J}_{1}^{m}(18)$ заменяется на $\left[D_{12}\right]_{2}=\left[D_{12}\right]_{1} /\left(1-\Delta_{12}\right)$, а для бародиффузионного фактора имеем

$$
\begin{gathered}
{\left[\alpha_{p}^{v}\right]_{2}=\left[\alpha_{p}^{v}\right]_{1}\left(1-\Delta_{p}\right)-\frac{2}{5}\left[\alpha_{T}\right]_{1}} \\
\Delta_{p}=\frac{2}{5}\left(\frac{m_{2}-m_{1}}{\bar{m}} x_{1} x_{2}\left[\alpha_{T}\right]_{1}+\frac{m_{2}+m_{1}}{\bar{m}} \frac{\Delta_{12}}{(6 / 5) C_{12}^{*}-1}\right) .
\end{gathered}
$$

Здесь $\left[\alpha_{T}\right]_{1}-$ термодиффузионный фактор, $\Delta_{12}-$ поправка второго приближения к коэффициенту бинарной диффузии. Выражения для $\left[\alpha_{T}\right]_{1}, \Delta_{12}$ и коэффициента $C_{12}^{*}$ можно найти в $[1,21,23]$.

В качестве иллюстрации характера зависимости бародиффузионного фактора от параметров частиц смеси и характера их взаимодействия приведем приближенное выражение для $\left[\alpha_{p}^{v}\right]_{2}$ в случае малого относительного различия масс и диаметров частиц для модели молекул твердых упругих сфер [3]:

$$
\left[\alpha_{p}^{\nu}\right]_{2}=1.41 \frac{m_{2}-m_{1}}{m_{2}+m_{1}}-1.26 \frac{d_{2}-d_{1}}{d_{2}+d_{1}}
$$

Как видно, при определенных соотношениях масс и диаметров рассеяния частиц бародиффузионный фактор может менять свой знак. Аналогичный характер поведения кинетического коэффициента известен для термодиффузии [1].

\section{Течение и диффузия газовой смеси в капилляре}

Полученные выше выражения могут быть использованы при анализе медленных течений газовой смеси в каналах под действием градиентов концентрации и давления. Рассмотрим в качестве примера течение бинарной газовой смеси вдоль оси $z$ в круглом цилиндрическом капилляре радиуса $R$. Следует иметь в виду, что для полного анализа некоторых физических эффектов, возникающих, например, в объемах, соединенных капиллярной трубкой (или системой капилляров), помимо выражения для усредненного по сечению канала молярного диффузионного потока $\bar{J}_{1 z}^{m}=n x_{1} x_{2}\left(\bar{u}_{1 z}-\bar{u}_{2 z}\right)$, оказывается необходимым также выражение для среднемолярного потока смеси $\bar{G}_{z}^{m}=n \bar{u}_{z}^{m}=n\left(x_{1} \bar{u}_{1 z}+x_{2} \bar{u}_{2 z}\right)$. Усредненные по сечению капилляра скорости компонентов смеси определяются при этом как

$$
\bar{u}_{\alpha z}=\left(2 / R^{2}\right) \int_{0}^{R} u_{\alpha z}(r) r d r .
$$

Напомним, что в рамках неравновесной термодинамики прерывных систем устанавливаются общие линейные феноменологические соотношения для $\bar{J}_{1 z}^{m}$ и $\bar{G}_{z}^{m}$, которые в случае изотермического течения смеси $(d T / d z=0)$ могут быть представлены в виде [18]:

$$
\begin{gathered}
\bar{J}_{1 z}^{m}=-n x_{1} x_{2}\left(\Lambda_{11} \frac{p}{T} \frac{d x_{1}}{d z}+\Lambda_{1 m} \frac{1}{T} \frac{d p}{d z}\right), \\
\bar{G}_{z}^{m}=-n\left(\Lambda_{m 1} \frac{p}{T} \frac{d x_{1}}{d z}+\Lambda_{m m} \frac{1}{T} \frac{d p}{d z}\right) .
\end{gathered}
$$

Для перекрестных коэффициентов соблюдается при этом принцип симметрии Онзагера $\left(\Lambda_{m 1}=\Lambda_{1 m}\right)$. 
Воспользуемся уравнением баланса импульса (14), записанным для компонента 1 смеси, которое для выбранной геометрии течения принимает вид:

$$
\frac{n_{1} n_{2} k T}{n\left[D_{12}\right]_{1}}\left(u_{1 z}-u_{2 z}\right)=-\frac{d p_{1}}{d z}-\frac{1}{r} \frac{\partial}{\partial r}\left(r \pi_{1 z r}\right) .
$$

Применение к уравнению (23) операции усреднения по сечению канала приводит к результату

$$
\bar{J}_{1 z}^{m}=-n\left[D_{12}\right]_{1}\left[\frac{d x_{1}}{d z}+x_{1} \frac{1}{p} \frac{d p}{d z}+\frac{2}{p R} \pi_{1 z r}(R)\right] .
$$

Известно, что характер течения и диффузии газовой смеси в каналах существенно зависит от значения числа Кнудсена $\mathrm{Kn}=\ell / R$, где $\ell-$ эффективная средняя длина свободного пробега молекул в смеси, которую можно определить как

$$
\ell=\left(\frac{2 k T}{\bar{m}}\right)^{1 / 2} \frac{\eta}{p}
$$

Для умеренно малых числах Кнудсена $(\mathrm{Kn}<0.10)$ значение парциального тензора вязких напряжений на стенке $\pi_{1 z r}(R)$ в выражении (24) может быть вычислено с помощью известных методов кинетической теории разреженного газа, которые учитывают поведение функции распределения молекул в пристеночном кнудсеновском слое (толщиной порядка нескольких длин свободного пробега молекул). В [26-28] эта задача решалась для случая течения и диффузии газовой смеси в плоском канале с использованием линеаризованных уравнений моментов метода Грэда (в сочетании с применением так называемого модифицированного метода Максвелла). Анализ течения в случае цилиндрической геометрии показывает, что с учетом малости толщины кнудсеновского слоя на стенке по сравнению с радиусом капилляра $(\ell \ll R)$ значение $\pi_{1 z r}(T)$ имеет тот же вид, что и в плоской задаче с заменой расстояния между плоскими стенками $d$ на радиус капилляра $R$. Тогда в пренебрежении членами, квадратичными по числу Кнудсена, интересующее нас выражение для случая полностью диффузного рассеяния молекул на стенке канала принимает вид

$$
\pi_{1 z r}(R)=-\frac{R}{4}\left(\frac{m_{1}^{1 / 2} x_{1}}{\left(m^{1 / 2}\right)_{x}}+\frac{\eta_{1}}{\eta}\right) \frac{d p}{d z},
$$

где $\left(m^{1 / 2}\right)_{x}=m_{1}^{1 / 2} x_{1}+m_{2}^{1 / 2} x_{2}$.

Подстановка (26) в (24) показывает, что вклад последнего члена в квадратных скобках (24) оказывается сравнимым со вторым членом, в результате выражение для $\bar{J}_{1 z}^{m}$ можно представить как

$$
\begin{gathered}
\bar{J}_{1 z}^{m}=-n\left[D_{12}\right]_{1}\left[\frac{d x_{1}}{d z}+\left[\bar{\alpha}_{p}\right]_{1} x_{1} x_{2} \frac{1}{p} \frac{d p}{d z}\right], \\
{\left[\bar{\alpha}_{p}\right]_{1}=\frac{1}{2}\left(\frac{m_{2}^{1 / 2}-m_{1}^{1 / 2}}{\left(m^{1 / 2}\right)_{x}}+\left[\alpha_{p}^{v}\right]_{1}\right) .}
\end{gathered}
$$

В следующем приближении в выражении (27) осуществляется замена $\left[D_{12}\right]_{1} \rightarrow\left[D_{12}\right]_{2}$ и $\left[\bar{\alpha}_{p}\right]_{1} \rightarrow\left[\bar{\alpha}_{p}\right]_{2}$, где $\left[\bar{\alpha}_{p}\right]_{2}$, получаемое в приближении тринадцати моментов $[27,28]$, записывается как

$$
\left[\bar{\alpha}_{p}\right]_{2}=\frac{1}{2}\left(\frac{m_{2}^{1 / 2}-m_{1}^{1 / 2}}{\left(m^{1 / 2}\right)_{x}}-\delta\right)+\frac{1}{2}\left[\alpha_{p}^{\nu}\right]_{2} .
$$

Здесь $\left[\alpha_{p}^{v}\right]_{2}$ определено выражением (20), а поправка $\delta$ принимает вид

$$
\begin{aligned}
\delta & =\frac{1}{5}\left\{\frac{m_{1}^{3 / 2} x_{1}+m_{2}^{3 / 2} x_{2}}{\left(m^{1 / 2}\right)_{x} \bar{m}}\left[\alpha_{T}\right]_{1}+\frac{m_{2}^{1 / 2}-m_{1}^{1 / 2}}{\left(m^{1 / 2}\right)_{x}} \frac{m_{1}+m_{2}}{\bar{m}}\right. \\
& \left.\times \frac{\Delta_{12}}{(6 / 5) C_{12}^{*}-1}\right\} .
\end{aligned}
$$

Новые значения бародиффузионного фактора $\bar{\alpha}_{p}$ по сравнению с $\alpha_{p}^{v}$ напрямую связаны с вкладом тонкого кнудсеновского слоя вблизи стенки [28]. В [26,28] приведены общие выражения $\left[\bar{\alpha}_{p}\right]_{2}$ для случая диффузнозеркальной модели рассеяния молекул на стенке канала при отличающихся значениях коэффициентов аккомодации импульса молекул $\kappa_{\alpha}$ для компонентов смеси на стенке. В частности, для газовой смеси с малым относительным различием масс, диаметров частиц и коэффициентов аккомодации [26]:

$$
\left[\bar{\alpha}_{p}\right]_{2}=1.27 \frac{m_{2}-m_{1}}{m_{2}+m_{1}}-0.60 \frac{d_{2}-d_{1}}{d_{2}+d_{1}}+1.89 \frac{\kappa_{2}-\kappa_{1}}{\kappa_{2}+\kappa_{1}} .
$$

Как видно из сравнения выражений (30) и (21), зависимости $\left[\bar{\alpha}_{p}\right]_{2}$ и $\left[\alpha_{p}^{\nu}\right]_{2}$ от различия масс частиц меняются при этом несущественно, однако численный коэффициент при относительной разнице в диаметрах частиц уменьшается примерно в два раза. Кроме того, возникает существенная зависимость $\bar{\alpha}_{p}$ от относительной разницы коэффициентов аккомодации импульса молекул на стенке. В табл. 1 представлены значения соответствующих бародиффузионных факторов, рассчитанных в первом и втором приближениях для ряда конкретных газовых смесей при $\kappa_{2}=\kappa_{1}=1$ с использованием параметров взаимодействия молекул для потенциала Леннард-Джонса, взятых из [23]. При этом компонент с индексом 1 соответствует более легким молекулам, концентрации компонентов смеси полагаются одинаковыми $\left(x_{1}=0.5\right)$. В первом и втором столбцах таблицы для сравнения приводятся также значения $\alpha_{p}^{m}$ (формула (4)) и кнудсеновского бародиффузионного фактора $\alpha_{p}^{K}$, выражение для которого обсуждается ниже (см. формулу (37)). Заметим, что для первых четырех смесей в таблице, отличающихся большим различием масс молекул компонентов, значения $\alpha_{p}^{K}, \alpha_{p}^{v}$ и $\bar{\alpha}_{p}$ относительно близки друг к другу. Следующие четыре смеси характеризуются относительно небольшим различием масс, но выраженной разницей в поперечниках рассеяния молекул. Это приводит к заметному различию в этих коэффициентах 
Таблица 1. Значения бародиффузионных факторов для газовых смесей

\begin{tabular}{c|c|c|c|c|r|r}
\hline Смесь & $\alpha_{p}$ & $\alpha_{p}^{K}$ & {$\left[\alpha_{p}^{v}\right]_{1}$} & {$\left[\alpha_{p}^{v}\right]_{2}$} & {$\left[\bar{\alpha}_{p}\right]_{1}$} & {$\left[\bar{\alpha}_{p}\right]_{2}$} \\
\hline $\mathrm{H}_{2}-\mathrm{Ar}$ & 1.8079 & 1.2663 & 1.3334 & 1.3774 & 1.2998 & 1.3340 \\
$\mathrm{He}-\mathrm{Ar}$ & 1.6357 & 1.0383 & 0.8925 & 0.9788 & 0.9654 & 1.0274 \\
$\mathrm{~N}_{2}-\mathrm{Ar}$ & 0.3512 & 0.1770 & 0.2374 & 0.2546 & 0.2072 & 0.2206 \\
$\mathrm{H}_{2}-\mathrm{N}_{2}$ & 1.7315 & 1.1540 & 1.1830 & 1.2402 & 1.1685 & 1.2119 \\
$\mathrm{D}_{2}-\mathrm{He}$ & 0.00065 & 0.00032 & 0.1891 & 0.1698 & 0.0947 & 0.0824 \\
$\mathrm{Ar}-\mathrm{CO}_{2}$ & 0.0968 & 0.0484 & -0.1286 & -0.1174 & 0.0401 & -0.0325 \\
$\mathrm{~N}_{2}-\mathrm{C}_{2} \mathrm{H}_{4}$ & 0.0015 & 0.0007 & -0.2201 & -0.2095 & -0.1097 & -0.1029 \\
$\mathrm{Ne}-\mathrm{C}_{2} \mathrm{H}_{4}$ & 0.3265 & 0.1644 & -0.3300 & -0.2766 & -0.0828 & -0.0469
\end{tabular}

и даже смене знака в значениях $\alpha_{p}^{v}$ и $\bar{\alpha}_{p}$ по отношению к значениям $\alpha_{p}$ и $\alpha_{p}^{K}$.

Выражение для среднемолярного потока смеси $\bar{G}_{z}^{m}$ при умеренно малых числах Кнудсена находится при решении задачи о течении газовой смеси в канале с учетом эффектов вязкого и диффузионного скольжения на границе смеси $[28,29]$. В случае круглого цилиндрического капилляра получаемый результат, соответствующий первому приближению, записывается в виде

$$
\bar{G}_{z}^{m}=-\frac{R^{2}}{8 \eta k T} p \frac{d p}{d z}\left(1+4 \sigma_{p} \mathrm{Kn}\right)+n\left[\sigma_{12}\right]_{1}\left[D_{12}\right]_{1} \frac{d x_{1}}{d z},
$$

где $\sigma_{p}$ и $\sigma_{12}-$ коэффициенты вязкого и диффузионного скольжения [28-30].

Сравнение выражений (27) и (31) с общими соотношениями (24) приводит к следующей связи коэффициентов

$$
\Lambda_{11}=\frac{T}{p} \frac{\left[D_{12}\right]_{1}}{x_{1} x_{2}}, \Lambda_{1 m}=\frac{T}{p}\left[\bar{\alpha}_{p}\right]_{1}\left[D_{12}\right]_{1},
$$

$$
\Lambda_{m 1}=-\frac{T}{p}\left[\sigma_{12}\right]_{1}\left[D_{12}\right]_{1}, \Lambda_{m m}=\frac{R^{2} T}{8 \eta}\left(1+4 \sigma_{p} \mathrm{Kn}\right) .
$$

Из выполнения принципа симметрии Онзагера $\left(\Lambda_{1 m}=\Lambda_{m 1}\right)$ при этом следует, что коэффициент диффузионного скольжения равен (с обратным знаком) бародиффузионному фактору: $\left[\sigma_{12}\right]_{1}=-\left[\bar{\alpha}_{p}\right]_{1}$. Конкретные расчеты подтверждают, что это соотношение оказывается справедливым в любом приближении используемых методов, в частности, $\left[\sigma_{12}\right]_{2}=-\left[\bar{\alpha}_{p}\right]_{2}$.

Область применения полученных выше результатов ограничена умеренно малыми числами Кнудсена (вязкое течение со скольжением). Рассмотрим также другой предельный случай течения газовой смеси в капилляре, когда выполняется условие $\mathrm{Kn} \gg 1$. Для этого так называемого режима свободно-молекулярного течения потоки каждого компонента смеси оказываются независимыми и определяются выражениями [31]:

$$
\begin{gathered}
n_{1} \bar{u}_{1 z}=-D_{1}^{K} \frac{d n_{1}}{d z}, n_{2} \bar{u}_{2 z}=-D_{2}^{K} \frac{d n_{2}}{d z} \\
D_{\alpha}^{K}=\frac{2}{3} R\left\langle v_{\alpha}\right\rangle b_{\alpha},\left\langle v_{\alpha}\right\rangle=\left(\frac{8 k T}{\pi m_{\alpha}}\right)^{1 / 2}, b_{\alpha}=\frac{2-\kappa_{\alpha}}{\kappa_{\alpha}} .
\end{gathered}
$$

Величина $D_{\alpha}^{K}$ носит название кнудсеновского коэффициента диффузии, $\kappa_{\alpha}-$ коэффициент аккомодации импульса молекул компонента $\alpha$ (для полностью диффузного рассеяния молекул на стенке $\left.\kappa_{\alpha}=1, b_{\alpha}=1\right)$. В случае бинарной газовой смеси, используя очевидные определения $\bar{J}_{1 z}^{m}$ и $G_{z}^{m}$ с помощью $\bar{u}_{1 z}$ и $\bar{u}_{2 z}$, и общие соотношения для них (22), для коэффициентов $\Lambda$ в этих соотношениях получаем

$$
\begin{gathered}
\Lambda_{11}=\frac{T}{p x_{1} x_{2}}\left(D_{1}^{K} x_{2}+D_{2}^{K} x_{1}\right), \\
\Lambda_{1 m}=\Lambda_{m 1}=\frac{T}{P}\left(D_{1}^{K}-D_{2}^{K}\right), \\
\Lambda_{m m}=\frac{T}{p}\left(D_{1}^{K} x_{1}+D_{2}^{K} x_{2}\right) .
\end{gathered}
$$

При этом молярный диффузионный поток $\bar{J}_{1 z}^{m}=$ $=n x_{1} x_{2}\left(\bar{u}_{1 z}-\bar{u}_{2 z}\right)$ записывается как

$$
\bar{J}_{1 z}^{m}=-n D_{12}^{K}\left[\frac{d x_{1}}{d z}+\alpha_{p}^{K} x_{1} x_{2} \frac{1}{p} \frac{d p}{d z}\right],
$$

где $D_{12}^{K}=D_{1}^{K} x_{2}+D_{2}^{K} x_{1}$ - обобщенный кнудсеновский коэффициент диффузии, а кнудсеновский бародиффузионный фактор $\alpha_{p}^{K}$ определяется как

$$
\begin{gathered}
\alpha_{p}^{K}=\frac{D_{1}^{K}-D_{2}^{K}}{D_{12}^{K}}=\frac{m_{2}^{1 / 2} b_{1}-m_{1}^{1 / 2} b_{2}}{\left(m^{1 / 2} b\right)_{x}}, \\
\left(m^{1 / 2} b\right)_{x}=m_{1}^{1 / 2} b_{2} x_{1}+m_{2}^{1 / 2} b_{1} x_{2} .
\end{gathered}
$$

Заметим, что исследование течений и диффузии в каналах при промежуточных числах Кнудсена $(\mathrm{Kn} \sim 1)$ основывается обычно на применении линеаризованного кинетического уравнения Больцмана для газовой смеси с модельным интегралом столкновений [32-35]. Получаемые для случая цилиндрического канала результаты оказываются при этом довольно громоздкими и требуют, как правило, использования численных методов решения. 


\section{Диффузионный бароэффект}

Полученные выше выражения для потоков могут служить основой для анализа ряда кинетических эффектов, проявляющихся при течении газовой смеси в капиллярах. Одним из них является возникновение нестационарной разности давлений $\Delta p(t)$ между объемами, соединенными пакетом узких капилляров или пористой мембраной толщиной $L$ и первоначально заполненными газовыми смесями разного состава, (диффузионный бароэффект [10,15-17]). Характер зависимости $\Delta p=p(L)-p(0)$ от времени определяется при этом соотношением диффузионного и гидродинамического потоков через капилляр. Перепад давлений и изменение концентрации компонентов смеси в объемах могут быть найдены из рассмотрения баланса числа частиц компонентов в объемах с учетом выражений для потоков (22). В частности, уравнение для определения разности давлений $\Delta p$ между одинаковыми объемами $V$ принимает вид

$$
\frac{d}{d t} \Delta p=\frac{2 k T}{V} S \bar{G}_{z}^{m}
$$

где $S$ - общая площадь сечения соединительных каналов. Для полного решения задачи уравнение (38) должно быть дополнено следующими из общих уравнений баланса уравнениями для изменения концентраций в объемах.

В кнудсеновском режиме течения $(\mathrm{Kn} \gg 1)$ времена релаксации для разности давлений и концентраций имеют один порядок величины, поэтому $\Delta p(t)$ достигает максимума и затем достаточно быстро спадает до нуля. Выражение для $\Delta p(t)$ в этом случае находится из условия баланса частиц в объемах для каждого из компонентов смеси при использовании выражений для кнудсеновских потоков (33). Для максимального перепада давлений $\Delta p_{\max }$ соответствующий результат принимает вид $[15,16]$ :

$$
\frac{\Delta p_{\max }}{p_{0}}=\left(\Delta x_{1}\right)_{0}\left[\exp \left(-\frac{\gamma \ln \gamma}{\gamma-1}\right)-\exp \left(-\frac{\ln \gamma}{\gamma-1}\right)\right],
$$

где $\left(\Delta x_{1}\right)_{0}=x_{1}(0)-x_{1}(L) \quad$ в $\quad$ момент $\quad$ времени $t=0$. Параметр $\gamma$ определяется при этом как $\gamma=\left(D_{1}^{K} / D_{2}^{K}\right)=\left(m_{2} / m_{1}\right)^{1 / 2}\left(b_{1} / b_{2}\right)$.

Для умеренно малых чисел Кнудсена (вязкое течение со скольжением) значения концентраций в объемах меняются значительно медленнее, чем давление, поэтому установившаяся максимальная разность давлений очень медленно спадает до нуля. В этом случае достаточно хорошим приближением для определения $\Delta p_{\max }$ является использование условия обращения в нуль потока смеси $\bar{G}_{z}^{m}$ в предположении, что разность концентраций в объемах остается приближенно равной своему начальному значению. Приравнивая нулю выражение для $\bar{G}_{z}^{m}(31)$ без учета поправки на вязкое скольжение,

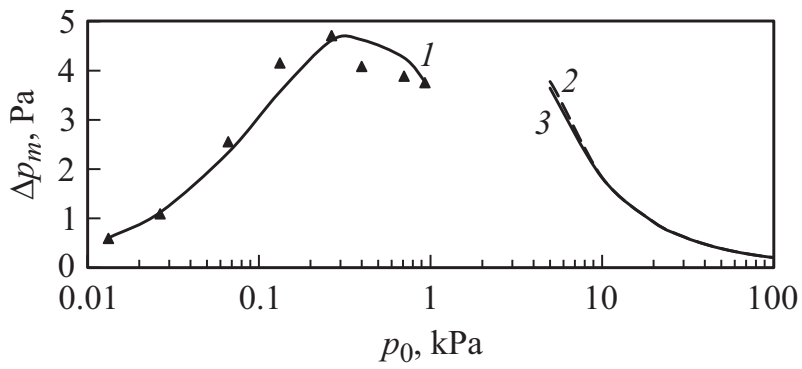

Рис. 1. Зависимости максимального перепада давлений $\Delta p_{m}$ от начального давления в объемах $p_{0}$ для смеси $\mathrm{Ar}-\mathrm{N}_{2}: \triangle-$ эксперимент [36], 1 - теоретический расчет [17], 2 - расчет (формула (41) - модель твердых сфер), 3 - расчет (формула (41) - модель Леннард-Джонса).

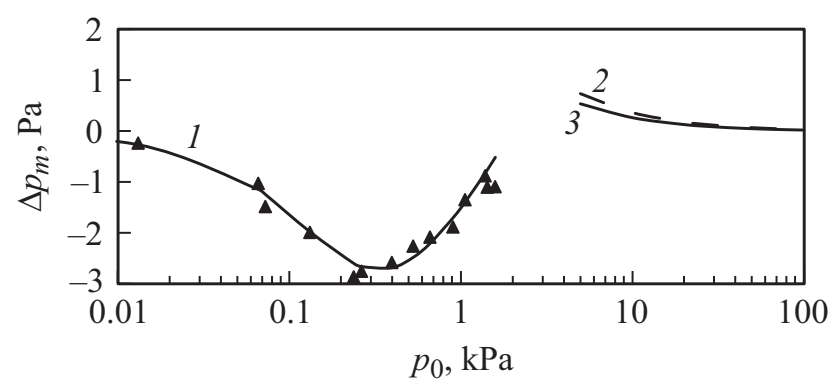

Рис. 2. Зависимости максимального перепада давлений $\Delta p_{m}$ от начального давления в объемах $p_{0}$ для смеси $\mathrm{CO}_{2}-\mathrm{Ar}$ : $\triangle$ - эксперимент [36], $1-$ теоретический расчет [17], 2 - расчет (формула (41) - модель твердых сфер), 3 - расчет (формула (41) - потенциал Леннард-Джонса).

которая в этом случае имеет следующий порядок малости по числу Кнудсена, приходим к соотношению

$$
d p=\left(8 \eta / R^{2}\right)\left[D_{12}\right]_{1}\left[\sigma_{12}\right]_{1} d x_{1},
$$

интегрирование которого по длине канала дает

$$
\Delta p_{\max }=\left(8\left[D_{12}\right]_{1} / R^{2}\right) \int_{x_{1}(0)}^{x_{1}(L)} \eta\left[\sigma_{12}\right]_{1} d x_{1} .
$$

Напомним, что $\left[\sigma_{12}\right]_{1}=-\left[\bar{\alpha}_{p}\right]_{1}$. Для более точных оценок на основе выражения (40) вместо коэффициентов диффузии и диффузионного скольжения первого приближения можно использовать их значения, рассчитанные во втором приближении $\left(\left[D_{12}\right]_{2}\right.$ и $\left.\left[\sigma_{12}\right]_{2}=-\left[\bar{\alpha}_{p}\right]_{2}\right)$, воспользовавшись, например, результатами [26-28]. В этом случае

$$
\Delta p_{\max }=-\left(8\left[D_{12}\right]_{2} / R^{2}\right) \int_{x_{1}(0)}^{x_{1}(L)} \eta\left[\bar{\alpha}_{p}\right]_{2} d x_{1} .
$$

В случае газовой смеси с малым относительным различием масс, диаметров частиц и коэффициентов аккомодации импульса значения $\eta$ и $\sigma_{12}$ слабо зависят от 


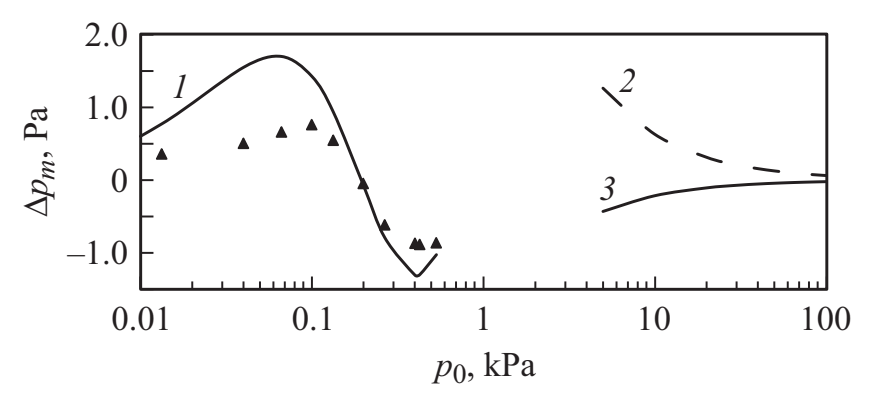

Рис. 3. Зависимости максимального перепада давлений $\Delta p_{m}$ от начального давления в объемах $p_{0}$ для смеси $\mathrm{C}_{2} \mathrm{H}_{4}-\mathrm{Ne}$ : $\triangle$ - эксперимент [36], $1-$ теоретический расчет [17], 2 - расчет (формула (41) - модель твердых сфер), 3 - расчет (формула (41) - потенциал Леннард-Джонса).

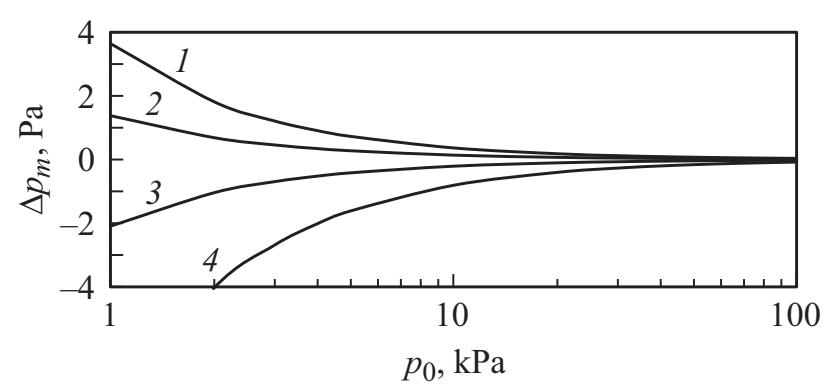

Рис. 4. Расчетные зависимости максимального перепада давлений $\Delta p_{m}$ от начального давления в объемах $p_{0}$ для смеси $\mathrm{C}_{2} \mathrm{H}_{4}-\mathrm{Ne}$ при различных значениях коэффициентов аккомодации импульса молекул на стенке; значение $\kappa_{1}=0.99$ для $\mathrm{C}_{2} \mathrm{H}_{4}$, значения $\kappa_{2}$ для $\mathrm{Ne}: 1-1.0 ; 2-0.98 ; 3-0.95$; $4-0.9$.

концентрации и могут быть вынесены за знак интегрирования. В этом случае для модели молекул-твердых упругих сфер, учитывая, что $\rho\left[D_{12}\right]_{2} / \eta=1.017(6 / 5)$, получаем

$$
\Delta p_{m} / p=4.99 \mathrm{Kn}^{2}\left[\bar{\alpha}_{p}\right]_{2} \Delta x_{1},
$$

где $\left[\bar{\alpha}_{p}\right]_{2}$ определяется выражением (30).

На рис. 1 представлено сравнение расчетных величин максимального перепада давлений $\Delta p_{\max }$ в функции от начального давления $p_{0}$ с результатами экспериментальных измерений диффузионного бароэффекта для смесей $\mathrm{Ar}-\mathrm{N}_{2}, \mathrm{CO}_{2}-\mathrm{Ar}$ и $\mathrm{C}_{2} \mathrm{H}_{4}-\mathrm{Ne}$ [36]. Теоретические кривые для промежуточных чисел Кнудсена взяты из работы [17], где величина эффекта рассчитывалась численным методом на основе выражений, получаемых в результате решения кинетического уравнения Больцмана с модельным интегралом столкновений в форме Мак-Кормака [37]. На тех же рисунках представлены результаты расчетов для области малых чисел Кнудсена на основе выражения (41), полученного в настоящей работе. Теоретические зависимости построены, начиная с давлений $p_{0}=5 \mathrm{kPa}$. Соответствующие этому давлению значения числа Кнудсена для указанных выше газовых смесей при $x_{1}=0.5$ равны соответственно $0.043,0.031$
Таблица 2. Параметры силового взаимодействия компонентов газовой смеси

\begin{tabular}{c|c|c|c|c}
\hline Газ & $\varepsilon / k, \mathrm{~K}$ & $\sigma, \mathrm{A}$ & $d, \mathrm{~A}$ & $\kappa$ \\
\hline $\mathrm{N}_{2}$ & 91.5 & 3.68 & 3.76 & 1.00 \\
$\mathrm{Ne}$ & 35.7 & 2.79 & 2.59 & 0.99 \\
$\mathrm{Ar}$ & 124.0 & 3.42 & 3.66 & 0.94 \\
$\mathrm{CO}_{2}$ & 190.0 & 3.99 & 4.63 & 1.00 \\
$\mathrm{C}_{2} \mathrm{H}_{4}$ & 205.0 & 4.23 & 4.36 & 1.00
\end{tabular}

и 0.043. В табл. 2 приведены использованные в расчетах кинетических коэффициентов параметры потенциала Леннард-Джонса [23] и модели жестких упругих шаров [1] для компонентов смесей. В последнем столбце таблицы представлены значения коэффициентов аккомодации импульса, принятые при расчетах [17].

К сожалению, экспериментальные результаты [36] не охватывают область умеренно малых чисел Кнудсена, где справедливы формулы настоящей статьи. Вместе с тем очевидно, что ход полученных в нашей работе теоретических зависимостей хорошо согласуется с кривыми, рассчитанными в [17] для промежуточных чисел Кнудсена. Обращает на себя внимание возможность смены знака эффекта в области малых чисел Кнудсена для смесей с относительно близкими значениями масс молекул компонентов смеси $\left(\mathrm{CO}_{2}-\mathrm{Ar}\right.$ и $\left.\mathrm{C}_{2} \mathrm{H}_{4}-\mathrm{Ne}\right)$. Это иллюстрируется поведением приведенных на рис. 3 и 4 расчетных кривых в случае смеси $\mathrm{C}_{2} \mathrm{H}_{4}-\mathrm{Ne}$, из которых следует, что знак эффекта может зависеть как от выбора модели взаимодействия молекул между собой (потенциал Леннард-Джонса, модель жестких упругих шаров), так и от соотношения коэффициентов аккомодации импульса молекул при их взаимодействии со стенкой.

\section{Эффект разделения}

Рассмотрим теперь другой эффект, который является в известной мере перекрестным по отношению к диффузионному бароэффекту $[10,13]$. Суть его состоит в возникновении скачка концентрации компонентов смеси в объемах, разделенных пакетом капилляров, если между объемами поддерживается постоянный перепад давлений $\Delta p=p_{0}-p^{\prime}$. Обозначим текущую концентрацию 1-го компонента в бинарной газовой смеси по длине капилляра, как $x_{1}$, а концентрации на входе и выходе из капилляра, как $x_{1}^{0}$ и $x_{1}$. Если потоки компонентов на выходе из капилляра являются чисто конвективными, то для установившегося течения смеси в капилляре имеем

$$
\bar{G}_{1}=\bar{G}_{z}^{m} x_{1}^{\prime}, \bar{G}_{2}=\bar{G}_{z}^{m}\left(1-x^{\prime}\right) .
$$

Молярный диффузионный поток 1-го компонента определяется соотношением $\bar{J}_{1 z}^{m}=\bar{G}_{1}-\bar{G}_{z}^{m} x_{1}$. С учетом (43) приходим тогда к уравнению вида

$$
\bar{G}_{z}^{m}\left(x_{1}^{\prime}-x_{1}\right)=\bar{J}_{1 z}^{m} \text {. }
$$


Для кнудсеновского режима течения газовой смеси $(\mathrm{Kn} \gg 1)$ при использовании выражений для $\bar{G}_{\alpha}(33)$ из (44) следует уравнение для изменения концентрации $x_{1}$ по длине капилляра

$$
p \frac{d x_{1}}{d p}+\left(x_{1}-x_{1}^{\prime}\right)+\alpha_{p}^{K}\left(x_{1}^{\prime}\right) x_{1}^{\prime}\left(1-x_{1}^{\prime}\right)=0 .
$$

Его решение приводит к следующему выражению для скачка концентрации $\Delta x_{1}=x_{1}^{\prime}-x_{1}^{0}$ :

$$
\Delta x_{1}=\frac{m_{2}^{1 / 2} b_{1}-m_{1}^{1 / 2} b_{2}}{\left(m^{1 / 2} b\right)_{x_{1}^{\prime}}} x_{1}^{\prime}\left(1-x_{1}^{\prime}\right)\left(1-\frac{p^{\prime}}{p_{0}}\right) \text {. }
$$

В другом предельном случае $\mathrm{Kn} \ll 1$ в уравнение $(44)$ можно подставить выражения для $\bar{J}_{1 z}^{m}$ и $\bar{G}_{z}^{m}$, полученные выше для области вязкого течения со скольжением. В пренебрежении членами, квадратичными относительно малой величины относительного эффекта разделения в этой области, приходим к уравнению вида

$$
\frac{d x_{1}}{d p}+(a p+b)\left(x_{1}-x_{1}^{\prime}\right)+\frac{1}{p} \bar{\alpha}_{p}\left(x_{1}^{0}\right) x_{1}^{0}\left(1-x_{1}^{0}\right)=0,
$$

где $\bar{\alpha}_{p}\left(x_{1}^{0}\right)$ определяется выражениями (28) или (29) (первое и второе приближение) при $x_{1}=x_{1}^{0}$. Коэффициенты $a$ и $b$ определены при этом как

$$
a=\frac{R^{2}}{8 \eta p_{0} D_{12}\left(p_{0}\right)}, \quad b=\frac{1}{2}\left(\frac{2 k T}{\bar{m}}\right)^{1 / 2} \frac{R \sigma_{p}}{p_{0} D_{12}\left(p_{0}\right)} .
$$

Решение (47) приводит к следующему выражению для разности концентраций на концах капилляра

$$
\begin{gathered}
\Delta x_{1}=\bar{\alpha}_{p} x_{1}^{0}\left(1-x_{1}^{0}\right) Y\left(p_{0}, p^{\prime}\right), \\
Y\left(p_{0}, p^{\prime}\right)=\int_{p^{\prime}}^{p_{0}} \frac{d p}{p} \exp \left[-\int_{p}^{p_{0}}(a p+b) d p\right] .
\end{gathered}
$$

Для газовой смеси с малым относительным различием масс, диаметров частиц и коэффициентов аккомодации импульса, в пренебрежении вкладом от вязкого скольжения и при условии $\Delta p / p_{0} \ll 1$, приближенное решение для $\Delta x_{1}$ можно представить в виде

$$
\Delta x_{1}=4.88 \mathrm{Kn}^{2}\left[\bar{\alpha}_{p}\right]_{2}\left[1-\exp \left(-\frac{0.208}{\mathrm{Kn}^{2}} \frac{\Delta p}{p_{0}}\right)\right],
$$

где $\left[\bar{\alpha}_{p}\right]_{2}$ определено выражением (30). Как и в случае диффузионного бароэффекта, эффект разделения в этой области течения пропорционален квадрату числа Кнудсена и может менять знак в зависимости от соотношений масс, диаметров частиц и коэффициентов аккомодации импульса для компонентов смеси.

К сожалению, число работ, посвященных экспериментальному исследованию эффекта разделения в капиллярах, весьма ограничено [38-41]. Как правило, этот эффект измеряется в условиях истечения газовой смеси в вакуум. Фактически это означает, что режим течения в капилляре меняется в широком диапазоне чисел Кнудсена, поэтому результаты настоящей работы, справедливые лишь для малых чисел Кнудсена, оказываются в этом случае неприменимыми для сравнения с экспериментом. Вместе с тем в $[38,39,41]$ обнаружена смена знака эффекта разделения при промежуточных значениях числа Кнудсена для смесей с близкими массами молекул компонентов $\left(\mathrm{C}_{2} \mathrm{H}_{4}-\mathrm{N}_{2}, \mathrm{CO}_{2}-\mathrm{Ar}, \mathrm{He}-\mathrm{D}_{2}\right)$, что согласуется с обсуждавшимися выше результатами по диффузионному бароэффекту.

\section{Заключение}

Как следует из проведенного выше анализа, характер диффузии в газовой смеси под действием приложенного градиента давления существенно зависит от особенностей течения смеси. Для медленных (вязких) течений смесей коэффициент бародиффузии существенно отличается от значений, получаемых методами обычной кинетической теории и термодинамики необратимых процессов, благодаря учету вязкого переноса импульса и влиянию кнудсеновских слоев в случае течения смеси в канале. Выражения для диффузионных потоков, получаемые в первом приближении методом баланса импульса и уточняемые в следующем приближении методами кинетической теории разреженного газа, позволяют провести корректный анализ ряда физических явлений, возникающих при течении газовых смесей через систему капилляров, соединяющих два объема. Сравнение с экспериментом показывает, что прецизионные измерения диффузионного бароэффекта и эффекта разделения в области промежуточных и малых чисел Кнудсена могут оказаться независимым эффективным инструментом для определения коэффициентов аккомодации импульса молекул отдельных компонентов смеси при их столкновении со стенкой.

Автор выражает благодарность Ю. Кагану за полезные обсуждения и В.А. Зазнобе за проведенные расчеты. Работа выполнена при финансовой поддержке Российского фонда фундаментальных исследований (проект № 18-0800211) и НИЯУ МИФИ в рамках программы повышения конкурентоспособности (контракт № 02.a03.21.0005, 27.08.2013)

\section{Список литературы}

[1] Чепмен C., Каулинг T. Математическая теория неоднородных газов. М.: ИЛ, 1960.510 с.

[2] Ландау Л.Д., Лифшии, Е.М. Гидродинамика. Теоретическая физика. Т. 6. М.: Наука, 1986. 734 с.

[3] Жданов В., Каган Ю., Сазыкин А. // ЖЭТФ. 1962. Т. 42. Вып. 3. С. 857-867.

[4] Потанин Е.П. // ЖТФ. 1983. Т. 53. Вып. 9. С. 1777-1781.

[5] Шапошников И., Гольдберг 3. // ЖЭТФ. 1952. Т. 23. Вып. 4. С. 425-429. 
[6] Дыхне А.М., Письменный В.Д., Пичугин В.В., Старостин А.Н., Таран М.Д. // ЖЭТФ. 1985. Т. 88. Вып. 6. C. $1976-1983$.

[7] Дьяков С.П. // ЖЭТФ. 1954. Т. 27. Вып. 3. С. 283-287.

[8] Борисевич В.Д., Борман В.Д., Сулаберидзе Г.А., Тихомиров А.В., Токманщев В.И. Физические основы разделения газовой смеси в газовой центрифуге. М.: Изд. дом МЭИ, 2011. $250 \mathrm{c}$.

[9] Жданов В.М., Карчевский А.И., Потанин Е.П., Устинов А.Л. // ЖТФ. 1979. Т. 49. Вып. 9. С. 1879-1885.

[10] Жданов В.М. // ЖТФ. 1967. Т. 37. Вып. 1. С. 192-197.

[11] Breton J.P. // Physica A. 1970. Vol. 50. P. 365-379.

[12] Zhdanov V.M. // Adv. Colloid Interface Sci. 1996. Vol. 66. P. 1-21.

[13] Villani S. Uranium Enrichment. Springer-Verlag. Berlin, Geidelberg, N. Y., 1976. 320 p.

[14] Изотопы: свойства, получение, применение / Под ред. В.Ю. Баранова. М.: Физматлит, 2005. Т. 1. 405 с.

[15] Waldmann L., Schmitt K.H. // Z. Naturforsch. A. 1961. B. 16a. P. 1343-1354.

[16] Суетин П.Е., Волобуев П.В. // ЖТФ. 1964. Т. 34. Вып. 6. C. $1107-1114$.

[17] Pan S., Storvik T.S. // J. Chem. Phys. 1992. Vol. 97. P. 26712681.

[18] Де Гроот C., Мазур П. Неравновесная термодинамика. М.: Мир, 1964. 456 c.

[19] Grad H. // Comm. Pure Appl. Math. 1949. Vol. 2. P. 331-407.

[20] Johnson M.H. // Phys. Rev. 1951. Vol. 82. P. 298.

[21] Жданов В.М. Явления переноса в многокомпонентной плазме. М.: Энергоиздат, 1982. 177 с.

[22] Франк-Каменецкий Д.А. Основы макрокинетики. Диффузия и теплопередача в химической кинетике, 4-е изд. Долгопрудный: Интеллект, 2008. 408 с.

[23] Гирифельдер Джс., Кертисс Ч., Берд Р. Молекулярная теория газов и жидкостей. М.: ИИЛ, 1961. 930 с.

[24] Потанин Е.П. // ЖТФ. 1984. Т. 54. Вып. 4. С. 803-807.

[25] Жданов В.М., Тирский Г.А. // ПММ. 2007. Т. 71. С. 794-81.

[26] Жданов В.М., Зазноба В.А. // ПММ. 1981. Т. 45. Вып. 6. C. $1063-1072$.

[27] Жданов В.М. // ПМТФ. 1982. № 2. С. 48-51.

[28] Zhdanov V.M. // Phys. Rev. E. 2017. Vol. 95. P. 033106.

[29] Loyalka S.K. // Phys. Fluids. 1971. Vol. 14. P. 2599.

[30] Ivchenko I.N., Loyalka S.K., Tompson R.V. // J. Vac. Sci. Technol. 1997. A15. C. 2375-2381.

[31] Kennard E.H. Kinetic Theory of Gases. NY.: McGrow-Hill, 1938.

[32] Shendalman L.H. // J. Chem. Phys. 1969. Vol. 51. P. 24832489.

[33] Почуев И.Д., Селезнев В.Д., Суетин П.Е. // ПМТФ. 1974. № 5. C. 37-41.

[34] Черняк В.Г. // ПМТФ. 1982. № 5. С. 51-58.

[35] Sharipov F., Kalempa D. // J. Vac. Sci. Technol. A. 2002. Vol. 20. N 3. P. 814-822.

[36] Lang H., Eger K. // Z. Phys. Chem. Neue Folge. 1969. B. 68. P. 130.

[37] McCormack F.J. // Phys. Fluids. 1973. Vol. 16. P. 2095-2105.

[38] Huggil J.A.W. // Proc. Roy. Soc. 1952. Vol. 212A. P. 123-136.

[39] Селезнев В.Д., Суетин П.Е., Смирнов Н.А. // ЖТФ. 1975. T. 45. C. $1499-1502$.

[40] Fain D.E., Brown W.K. Rarefied Gas Dynamics, Proc. X Intern. Symp. Plenum Press, 1976. Ed. J.L. Potter. Vol. 1. P. 65-78.

[41] Selesnev V., Porodnov B., Akinshin V., Surguchev V., Tarin A. Rarefied Gas Dynamics, Proc. XIII Intern. Symp. Plenum Press, 1985. Ed. A.K. Rebrov. Vol. 2. P. 1341-1348. 